\title{
Research on two Typical Estimation Algorithms of Doppler Frequency
}

\author{
Guodong Xu \\ Research center of Satellite Technology, Harbin \\ Technology Institute
}

Pengfei Li

Research center of Satellite Technology, Harbin

Technology Institute

Limin Dong

Research center of Satellite Technology, Harbin Technology Institute

\begin{abstract}
The accuracy of Doppler mean frequence estimation is vital in some related areas. In this paper, two typical estimation algorithms for mean frequency in time domain are discussed. Based on the mathematics derivation of different methods, the conclusion could be obatained that the precision was mostly influced by the amount of the sampling, signal-tonoise ratio (SNR), and the frequency of sampling. Then they were detailed analyzed and simulated in computer. The results of the simulations primarily indicate that the performance of complex-correlation algorithm is more advantageous than cross zero point algorithm in the same configeure condition. However, the complexity of its process is increased largely.
\end{abstract}

Keywords-Frequency estimation,Cross zero point,Complexcrrelation

\section{INTRODUCTION}

The Doppler method has widely be used to measure the velocity of object untouched, even detect vessel disease[1]. The frequency of the resulting signal will changed in the process of the ultrasonic transmission, owing to the relative movement occurred. The velocity of the object is related with the frequency of Doppler echo signal, not with the others, from the formula[2]. In fact the Doppler signal from undisturbed flow is random, with Gaussian amplitude probability density function[3]. There has been substantial prior work in the use of the fast Fourier transform to estimate the frequency of signal in additive Gaussian noise. These techniques, however, require a long observation period to resolve the frequency of the signal spectrum, it is unsuitable for the real-time of applications. Cross zero point algorithm $(\mathrm{CZ})$ and Complex-correlation algorithm(CC) are typical resolvent in time domain. The performance of a mean Doppler frequency estimator depends on several parameters such as SNR, the spectral distribution of Doppler shift frequencies, and the frequency response of signal processing hardware[4][5].

The aim of this paper was how to use these two algorithms in application on the base of derivation of them, and which one was selected according to the result shown in simulation, in which the amount of the sampling, sianal-tonoise ratio(SNR), and the frequency of sampling were taken into account.

\section{DESCRIPTION OF CROSS ZERO POINT ALGORITHM}

Cross zero point algorithm is not complex, and was achieved completely with Schmitt trigger circuit[6]. Its theory is shown in Figure.1. The ultrasonic Doppler signal from a acoustical-electrical transducer is passed through a low-pass filter to remove high-frequency noise, and sent, after amplification, to a AD circuit. The observed Doppler frequency is

$$
f_{d}=\frac{1}{T}=\frac{1}{t(n)-t(m)}=\frac{1}{(n-m) T n}
$$

where $T n$ is sample period, $n(m)$ is the times of the sampling value $X(n)(X(m))$. The process of the derivation of this formula was based on the real zero point replaced by $X(n)(X(m))$, in thoery, the precision will be higher as the sample period is shorter. The fact is not as and Figure. 2 show its cause, the precision in longger sample period will be better than the shorter one.

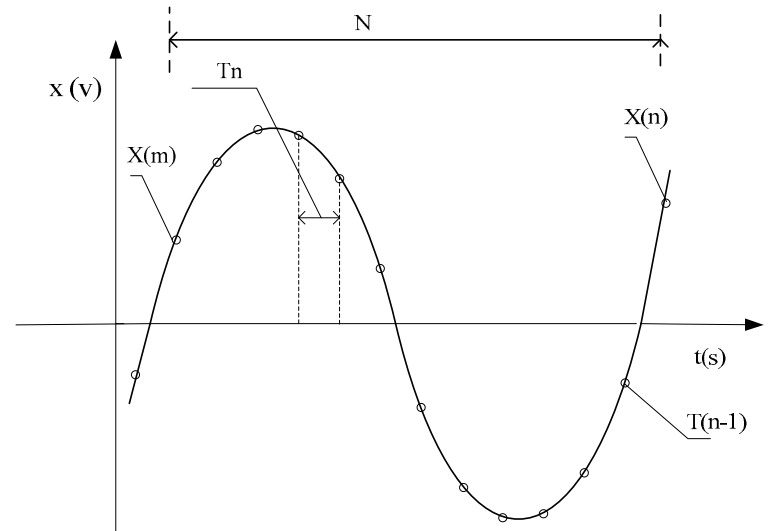

Figure 1. Theory of cross zero point algorithm

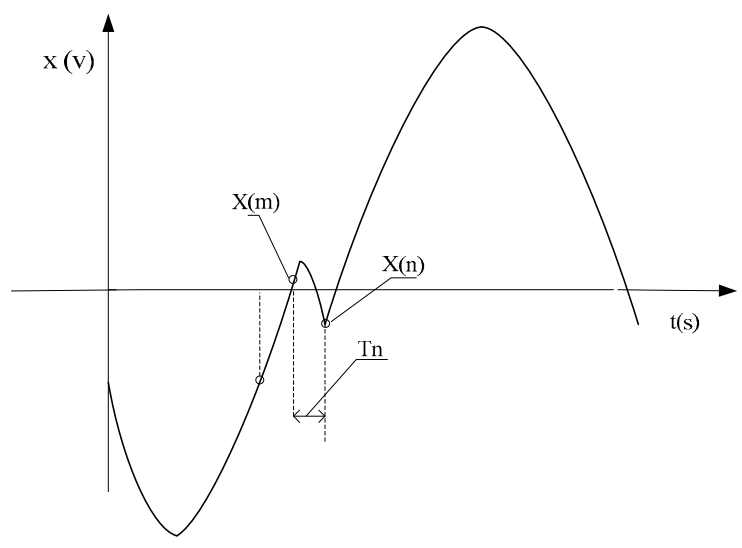

Figure 2. Influence of noise 


\section{THE DERIVATION OF COMPLEX-CORRELATION ALGORITHM}

It is assumed that the Doppler echo signal returned from object through a sample volume can be defined as,

$$
e(t)=\operatorname{Re}\left\{z(t) e^{j 2 \pi f_{0} t}\right\}
$$

Where Re represents the ensemble of real numbers of $e(t)$, $f_{0}$ is the frequency of Doppler echo signal. The in-phase and quadrature components of the siganls can be resolved separately,

$$
z(t)=x(t)+j y(t)
$$

By applying Connexions, and the Connexions's function of $z(t)$ can be stated as:

$$
R(T, t)=\operatorname{Re}+j R m
$$

where

$$
\begin{aligned}
& \mathrm{R}_{e}=\int_{t}\left[x\left(t^{\prime}\right) x\left(t^{\prime}-T\right)+y\left(t^{\prime}\right) y\left(t^{\prime}-T\right)\right] d t^{\prime} \\
& \mathrm{R}_{m}=\int_{t}\left[y\left(t^{\prime}\right) x\left(t^{\prime}-T\right)-x\left(t^{\prime}\right) y\left(t^{\prime}-T\right)\right] d t^{\prime}
\end{aligned}
$$

Eq.4 can be written as in index form,

$$
R(T, t)=|R(T, t)| e^{j \varphi(T, t)}
$$

where

$$
\begin{aligned}
|R(T, t)| & =\sqrt{R_{e}^{2}(T, t)+R_{m}^{2}(T, t)}=A(T, t) \\
\varphi(T, t) & =t^{-1} \frac{R_{m}(T, t)}{R_{e}(T, t)}
\end{aligned}
$$

Because $|R(T, t)|$ is even function, $\varphi(T, t)$ is odd function, nd in the condition of $T=0$, the derived function of Eq.7 can be writen as:

$$
\left.R^{\prime}(T, t)\right|_{T=0}=j|R(0, t)| \varphi^{\prime}(0, t)
$$

The mean frequency of the Doppler power spectrum can be defined as:

$$
\omega_{A}=\frac{\frac{1}{2 \pi} \int_{-\infty}^{\infty} \omega s(\omega) d \omega}{\frac{1}{2 \pi} \int_{-\infty}^{\infty} s(\omega) d \omega}
$$

By applying Wiener-Khintchine theorem, $R(T, t)$ can be discribed as:

$$
R(T, t)=\frac{1}{2 \pi} \int s(\omega, t) e^{j \omega T} d \omega
$$

where $s(\omega, t)$ is power spectral density, The derived function of Eq.12 which is in the condition of $T=0$ combined to Eq. 10 and Eq. 12 .

$$
\omega_{A}=\varphi^{\prime}(0, t)
$$

$\varphi^{\prime}(0, t)$ can be discribed in limiting form as:

$$
\omega_{A}=\varphi^{\prime}(0, t)=\lim _{T \rightarrow 0} \frac{\varphi(T, t)}{T}
$$

It is assumed that the Doppler signal which is in additive noise can be defined as:

$$
z(t)=S_{n}+N_{n}=\left(x_{n}+j y_{n}\right)+j\left(N x_{n}+j N y_{n}\right)
$$

where $S_{n}$ is the sequence of frequency of Doppler signal, and $N$ is the sequence of noise. so Eq.14 can be written in discrete notation as:

$$
\omega_{A}=\frac{\operatorname{tg}^{-1}\left[\frac{\sum_{n=1}^{N}\left(x_{n} y_{n+1}-x_{n+1} y_{n}\right)}{\left.\sum_{n=1}^{N}\left(x_{n} x_{n+1}+y_{n+1} y_{n}\right)\right]}\right.}{T}
$$

\section{SIMULATION AND DISCUSSION}

The performance of a estimator of the mean Doppler frequency is influenced by several parameters such as the amount of the sampling components, SNR, and the frequency of sampling. A simulation is designed to evaluate the two algorithm under a variety of special condition. It is assumed that there is a finite number of discrete Doppler signal components has been obtained by sampled in the simulation. The noise present in the Doppler signal simulated is defined as having a uniform frequency distribution, and the uniform distributed amplitude weighting with a mean of zero.

The result simulated of the mean frequency estimator using $\mathrm{CZ}$ algorithm is plotted in Figure.3. The condition of the simulation is that the frenquency of Doppler signal is foregone, it is $54 \mathrm{kHz}$ (Doppler shift frequency is $4 \mathrm{kHz}$ ) and the period of simpling is 30 us. The mean frequency estimate restrained the mean frequency of the signal after $10 \mathrm{~dB}$. As the SNR was increased from 0.5 to 10 , the mean frequency estimate converged toward the frequency as defined. The more components is beneficial for reducing probable error, because the simulation curve in the upper restrained to a greater scope. There are a certain amount of random errors in condition of even $100 \mathrm{~dB}$.
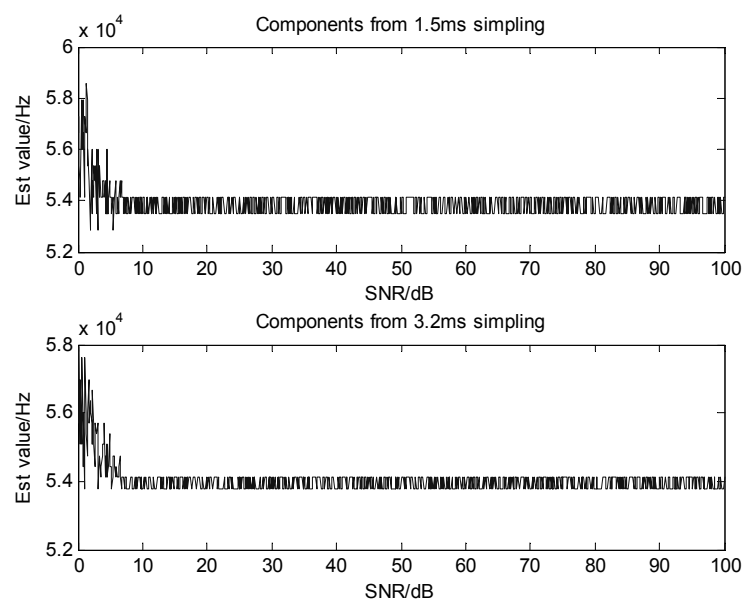

Figure 3. Influenced by SNR using CZ Algorithm 
Figure. 4 is a plot of Doppler shift frequency estimate influenced by frequency of simple. The condition of the simulation is under the same SNR which is $10 \mathrm{~dB}$, and Doppler shift frequency is $4 \mathrm{kHz}$. The mean Doppler shift frequency estimate using $\mathrm{CC}$ algorithm is $4 \mathrm{kHz}$ without any apparent estimate bias. As the simpling period increased from $20 \mathrm{us}$ to $60 \mathrm{us}$, the simulation curve using $\mathrm{CZ}$ algorithm is closer to $4 \mathrm{kHz}$, outside the area which from $20 \mathrm{us}$ to $60 \mathrm{us}$, however, the estimate bias is very great, the cause of this was analysed in the second section.

Figure.5 and Figure.6 are plots of Doppler shift frequency estimate influenced by SNR and the time of simple separately. The first one's condition is as same as Figure.3's. They show that the performance of esimator using $\mathrm{CC}$ algorithm is more advantageous than using $\mathrm{CZ}$ algorithm, and the bias was controlled in $2 \%$ easily.
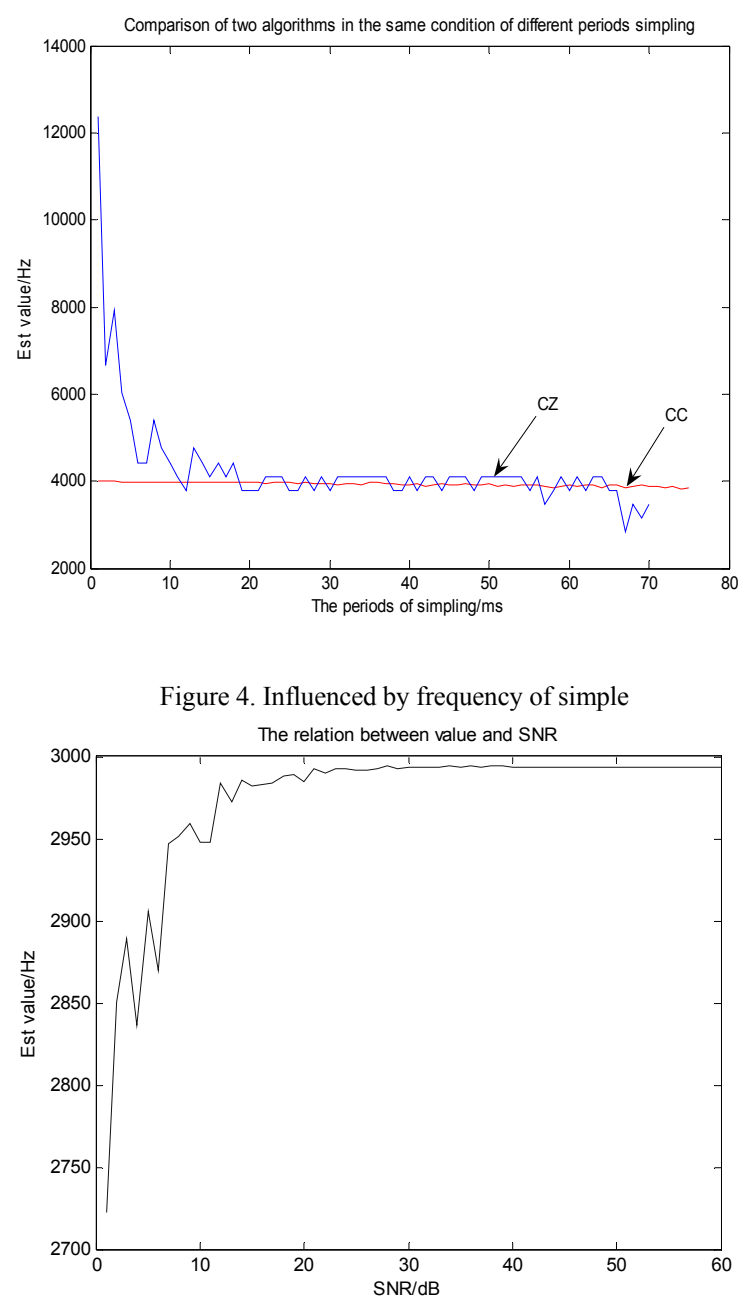

Figure 5. Influenced by SNR using CC Algorithm

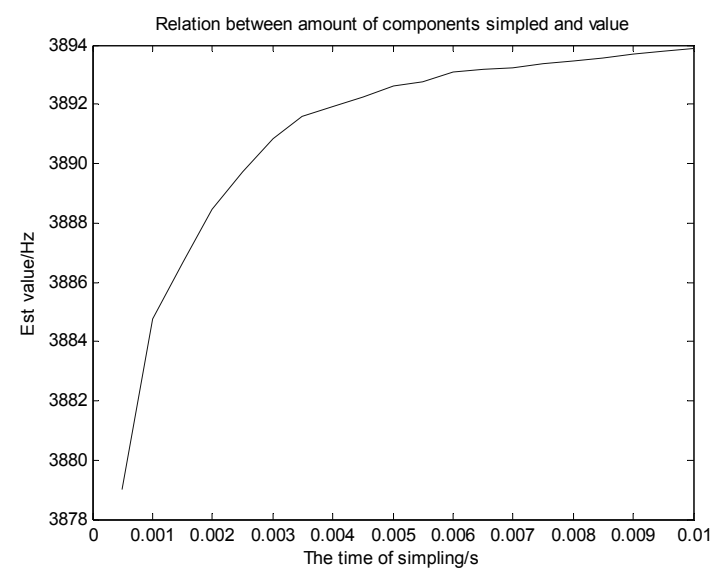

Figure 6. Influenced by the time of simple using CC Algorithm

\section{SUMMARIES}

Based on the mathematics dervation and simulation of the two methods, in this paper, the conclusions have been obtatained that the precision was influced by the amount of the sampling, signal-to-noise ratio(SNR), and the frequency of sampling, and were detailed analyzed. These simulation results demonstrate that $\mathrm{CZ}$ algorithm and $\mathrm{CC}$ algorithm have their own scope separately, and the second one is more advantageous than the first one in the same configeure condition, but the complexity of its process is increased largely.

\section{REFERENCES}

[1] CAC Bastos; PJ Fish. A Doppler signal simulator]. Clin. Phys. Physiol. Meas, 1991, PP:177-183

[2] WANG Xin-xiao; HUANG Jian-guo; ZHANG Qun-fei; YAN Wei. Efficient Simulation Method of Underwater Target Echo Based on Complex Envelopes. JOURNAL OF SYSTEM SIMULATION, 2005.11, PP:2852-2854

[3] Nihal F. Giiler. A Time Domain Mean Frequency Estimation Algorithm of Two-Dimensional Real-Time Doppler Imaging System. Journal of Medical Systems, 1995, PP:405-411

[4] XU Shi-jun; XU Wei-jie. A hardware efficient implementation of frequency estimation based on complex correlation method. TechnicalAcoustics, 2009.4 PP:180-183

[5] Wen Jing; Wen Yu-mei; Li Ping. Adaptive noise cancellation method based on error whitening criterion. Chinese Journal of Scientific Instrument. 2010.8 PP:1693-1699

[6] Ichiro Tai; Ken-ichi Hasegawa; Akira Sekiguchi. Digital frequency meter for burst-like laser Doppler signals. Scientific Instruments[J], 1997, PP:811-813 